\title{
Friendly Partner System of Poker Game with Facial Expressions
}

\author{
K. Ohsone, Student Member, IEEE and T. Onisawa, Member, IEEE
}

\begin{abstract}
This paper aims at the construction of a partner system with facial expressions, which plays a seven-card stud poker game with a human player against an opponent player. If a human player needs some advice on a game, the partner system has various facial expressions according to the current situation. Fuzzy theory is applied to decision-making part in the partner system and neural network is applied to the facial expressions part in the partner system. This paper also performs subject experiments to confirm the friendliness of the presented partner system with facial expressions, where each subject plays games with/without facial expressions.
\end{abstract}

\section{INTRODUCTION}

In our daily life we are often confronted with decision-making problems under uncertain situations. Although we think of many alternatives or various different opinions under such situation [1], it is usually difficult to solve them completely. Therefore, we often get other perspectives or hints to solve problems by exchanging opinions among others or by taking advice from others. An adviser with ripe experience may give better advice on decision-making problems. Exchanging opinions or taking advice may lead to some solution that cannot be found by oneself or to some other understanding on problems. From this point of view, there are many studies on decision support systems $[2,3,4]$. However, these systems only support users' decision, and do not consider decision-making program from the viewpoint of cooperation with human users.

This paper considers a cooperation of human with a computer in decision-making problems. As an example of the problems, this paper focuses on a seven-card-stud poker game, a kind of a game with imperfect information [5]. In such a game, situations estimate is usually difficult because of uncertainty of situations. There are also some studies about negotiation agent in a game, e.g., a MONOPOLY game [6], or poker playing systems $[7,8,9]$.

By the way, it is said that facial expressions give much information on feelings in human-to-human interaction [10]. In other words, facial expressions are more important than other nonverbal information in human-to-human communication. Therefore, even in the study on a poker game there is a study on a poker agent with face [11].

This paper aims at the construction of not a mere poker

Keisuke Ohsone and Takehisa Onisawa are with Graduate School of Systems and Information Engineering, University of Tsukuba, 1-1-1, Tennodai, Tsukuba, 305-8573 Japan (corresponding author to provide phone: 029-853-6188; fax: 029-853-6471;

e-mail: kfukuda@fhuman.esys.tsukuba.ac.jp;

e-mail: onisawa@iit.tsukuba.ac.jp). playing system but a partner system, which has a discussion with a human player on game strategy in a seven-card stud poker game cooperating with the player. Furthermore, this paper also introduces facial expressions to the partner system because the partner system is expected to become friendly by the use of not only linguistic expressions but also facial expressions. The presented system is based on the authors' previous seven-card-stud poker game playing system $[12,13]$, and is extended into from our previous partner system [14]. In this paper friendliness of the presented partner system using linguistic and facial expressions is compared with that of the previous partner system using only linguistic expressions.

The organization of the paper is as follows. In Section 2 a seven-card-stud poker game used in this paper is explained. Section 3 presents the partner system consisting of the decision-making module, the facial expressions module, and the cooperation module. Section 4 mentions subject experiments performed in order to show the usefulness/friendliness of the presented partner system with facial expressions and their results. Final section shows conclusions of this paper.

\section{SEVEN-CARD STUd POKER}

\section{A. Stud Poker}

A stud poker game is different from a draw poker one. And although a stud poker game has various kinds of game variations, face-down and face-up cards are dealt to each player in every stud poker game. The card dealt face down is called a hole card which only a card owner can see. On the other hand the card dealt face up is called an up card which all players can see.

\section{B. Seven-Card Stud Poker}

This paper considers the situation in which a human player and the partner system play a poker game together against the previous poker playing system $[12,13]$ as an opponent player. This section describes rules of a seven-card-stud poker game used in this paper.

After both players (a human player and the playing system) pay 5 points as the ante, two hole cards and one up card are dealt to each player, and the first betting round is started. The second player must bet at least the same amount of bet as the first player's one, where one player can raise the betting point only once at each betting round, and the upper limit of the betting points is assumed to be the total points betted so far. When both players' betting points become even, a next card is dealt to each player and the next betting round is started. The 
fourth, the fifth and the sixth cards are face-up cards, and the seventh card is a hole card. After all cards are dealt to and betting rounds are ended, each player makes a decision which hole card is revealed. Each player reveals one hole card and the betting round is started. If any players still don't fold a game, the procedure of revealing hole cards and betting round are continued. Bluff strategy or slow play is also often taken in the procedure of revealing hole cards. If both players continue the game after the final betting round, they show their final hole cards each other, which is called Showdown, and the player having a stronger poker hand with five cards out of seven is a winner of the game.

\section{Poker Hands}

This paper considers 10 sorts of poker hands including no pair (high card). Scores of these hands are defined as shown in TABLE I . In this paper, scores are simply defined as 10 scales from 0 to 9 because the linear scale is simple.

TABLE I

SCORE OF POKER HANDS

\begin{tabular}{|c|c|c|c|}
\hline Poker Hands & Scores & Poker Hands & Scores \\
\hline Nothing & 0 & Flush & 5 \\
\hline One Pair & 1 & Full House & 6 \\
\hline Two Pairs & 2 & Four of a Kind & 7 \\
\hline Three of a Kind & 3 & Straight Flush & 8 \\
\hline Straight & 4 & Royal Straight Flush & 9 \\
\hline
\end{tabular}

\section{PARTNER SYSTEM}

\section{A. Relationship between System and Players}

A human player plays a poker game in cooperation with the partner system against an opponent player. It is assumed that when the partner system is called by a human player according to game situations, the system presents system's strategy to a human player. Furthermore, when a human player has some questions, the partner system answers these questions. It is also assumed that the partner system has same information as the one a human player does. Fig. 1 shows the decision-making procedure of the partner system, which has the decision-making module, the cooperation module, and the facial expressions module.

\section{B. Decision-making Module}

The decision-making module of the partner system consists of six parts, winning rate estimation, assessment of superiority/inferiority, decision-making to fold or not, decision-making on betting points, choice of a card to be revealed and modification of fuzzy rules.

\section{1) Winning Rate Estimation}

In order to estimate the winning rate of a partner player, the system suppositionally assigns possible combination of cards to human and opponent players' hole cards and to their up cards dealt from now on. The system makes a judgment on human partner player's winning or losing. The system repeats this trial and counts the number of the partner player's winning. Let this repeating trial times and the number of human partner player's winning be $N_{\text {times }}$ and $N_{\text {win }}$, respectively. The winning rate is defined by

$$
P_{\text {win }}=\frac{N_{\text {win }}}{N_{\text {times }}} \text {. }
$$

In this paper, $N_{\text {times }}$ is fixed to be 1000 .

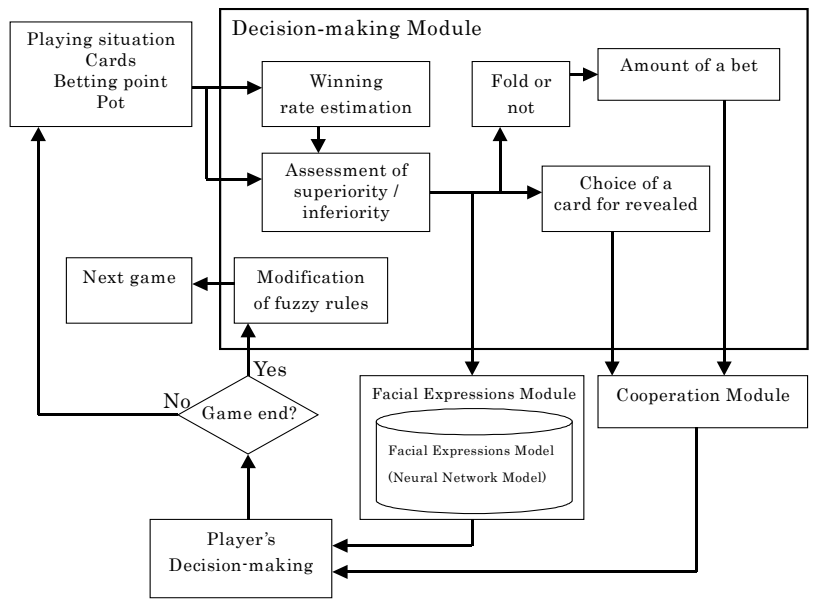

Fig. 1. Decision-making procedure of partner system

2) Assessment of Superiority/Inferiority

The relative strength of the human partner player's poker hand to the opponent player's poker hand is defined by

$$
\text { expect }=2\left(P_{\text {win }}-0.5\right)
$$

The expect means that the larger the expect is, the higher the possibility of the superiority of a human player's hand is. In this paper the obtained expect is adjusted by fuzzy inference using fuzzy rules considering the tendency of opponent player's strategy, betting points and the number of turn. TABLE II shows an example of fuzzy rules at the early game stage, where the turn is normalized by 7 , which is the maximum number of betting round times in a seven-card stud poker game, and betting points are normalized by the pot. As TABLE II shows, the fuzzy rules have parameter values $t_{i j} \in[0,100]$ called the tendency values. The tendency value means that if $t_{i j}$ is larger than 50, an opponent player tends to take aggressive strategy, and if $t_{i j}$ is smaller than 50, an opponent player tends to take passive strategy [13]. The initial values of all tendency values are defined to be 50 and the tendency values are changed every game according to the opponent player's strategy.

The fuzzy inference using these fuzzy rules is performed as follows. Let the result of fuzzy inference using the Positive consequent part and that using the Negative consequent part be $C_{\text {positive }}$ and $C_{\text {negative }}$, respectively, where the min-max inference method and the center of gravity are used in the fuzzy inference [15].

$$
\text { infer }=C_{\text {positive }} \times\left(\frac{t_{i j}}{100}\right)+C_{\text {negative }} \times\left(1-\frac{t_{i j}}{100}\right) \text {. }
$$


And expect is adjusted in the range of $[0,1]$ using the result of the fuzzy inference (3) by

$$
\text { result }=\left\{\begin{array}{r}
\text { expect }+(1-\text { expect }) \times \text { infer } \\
(0 \leq \text { infer } \leq 1) \\
\text { expect }+(\text { expect }+1) \times \text { infer } \\
(-1 \leq \text { infer } \leq 0)
\end{array}\right.
$$

The result is the assessment result of the superiority/ inferiority of a human player's poker hand.

TABLE II

FUZZY RULES FOR ASSESSMENT OF SUPERIORITY/INFERIORITY AT EARLY GAME

\begin{tabular}{|c|c|c|c|c|}
\hline \multicolumn{2}{|c|}{$\begin{array}{c}\text { Adjustment degree } \\
\text { (Turn is Early) }\end{array}$} & \multicolumn{3}{|c|}{ Opponent's bet } \\
\cline { 3 - 5 } & & Few & Middle & Much \\
\hline \multirow{3}{*}{ Weak } & Negative & Negative & Negative \\
& $t_{11}$ & $t_{12}$ & $t_{13}$ \\
Strength & & Positive & Positive & Positive \\
\cline { 3 - 5 } & & Negative & Negative & Negative \\
& \multirow{2}{*}{ Strong } & $t_{21}$ & $t_{22}$ & $t_{23}$ \\
& & Positive & Positive & Positive \\
\hline
\end{tabular}

3) Decision to Fold or Not

From the first through the fifth turns the partner system has the following decision procedures. When the system assesses the partner player's hand at superiority, the partner system advises to continue a game. When the partner system assesses the partner player's hand at inferiority, the partner system makes a decision by the fuzzy inference whether a partner player should fold a game or not. For example, the partner system has a rule; if opponent's betting points is large and the number of turn is large then tendency to fold is strong. If the fuzzy inference result $(\in[0,1])$ is larger than a uniformly random generated number $(\in[0,1])$, the partner system makes a decision to fold a game.

In the sixth and the seventh turns, a poker hand is already determined because seventh card is already dealt. However, even if the hand strength is assessed at inferiority, there is sometimes a case where it is possible to take bluff strategy. The presented system decides whether bluff strategy is taken or not according to the following procedures.

In order to choose a hole card to be revealed, the potentiality is obtained by the following way. Assuming that one partner player's hole card is revealed, possible combinations of cards are assigned to remaining hole card(s). The expected value of the strength of possible poker hands is obtained by

$$
\text { potential }=\sum_{i=1}^{m} u_{i} p\left(u_{i}\right),
$$

where $m$ is the number of partner's possible poker hands, $u_{i}$ is the score of the possible hand shown in TABLE I, and $p\left(u_{i}\right)$ is probability of $u_{i}$. This expected value is called potential in this paper. In the same way, the system obtains the expected value of opponent player's hand called Opp _ potential .

If Eq. (6) is satisfied, the partner system decides not to fold and to take a bluff strategy. This parameter value is obtained by previous work [13].

$$
\text { potential } \geq O p p_{\text {_ }} \text { potential }+1.3
$$

\section{4) Decision on Betting Points}

Let the upper limit and the lower limit of betting points be upper and lower, respectively, where these limits are dependent on the betting round and are provided by the poker game rules. In this paper the upper limit is assumed to be the total points betted so far and the lower limit is assumed to be 5 points. The bet points are obtained by

$$
C_{\text {bet }}=\text { lower }+ \text { a }(\text { upper }- \text { lower }),
$$

where $a$ is defined by

$$
a=\frac{\text { result }+1}{2}+\text { noise }
$$

where noise is a normally distributed random number with the mean value 0 and the variance 0.1 . If bluff strategy is considered by the system, $C_{b e t}$ in Eq. (7) is obtained using $a$ defined by

$$
a=\frac{(\text { potential } / 9)+1}{2},
$$

where potential is obtained by Eq.(5) and is normalized by 9 , i.e., the maximum score of poker hands defined by Table 2 . This means that the system bets large points in order to make the partner player's hand look strong using the bluff strategy.

5) Choice of a Card to be Revealed

In the fifth and the sixth turns, after the betting round is finished, each player chooses one hole card to be revealed and turns it face up.

When the system assesses a partner's hand at superiority and the hand is already shown in face-up cards, a card to be revealed is chosen at random. When the system assesses a partner's hand at superiority and the hand is hidden in hole cards, the system advises to reveal the card at the rate of $80 \%$, of which potential is the lowest. The system advises to reveal the card at the rate of $20 \%$, of which potential is the second lowest.

When the system assesses a partner's hand at inferiority, the system advises to reveal the card of which potential is the highest.

6) Modification of Fuzzy Rules

All cards are shown at the game end and both players' 
hands are revealed. Every one game the modification part of fuzzy rules estimates the change of the tendency value considering the difference between the estimated relative hand strength and actual hand strength by using the fuzzy inference. The modification is performed for the fuzzy rule of which satisfaction degree is the highest in the assessment of superiority/inferiority. The fuzzy rules for modifying the fuzzy rules for assessment of superiority/inferiority have the following form of statement. (1) If the estimated relative poker hand strength is lower than the actual poker hand strength, then the tendency value is changed to be small. (2) If the estimated relative poker hand strength is higher than the actual poker hand strength, then the tendency value is changed to be large. (3) If the estimated relative poker hand strength and the actual one are even, then the tendency value is not changed.

The tendency value is actually changed considering the last five estimated changing values of the tendency value and the latest changing value in order to consider opponent player's tendency in a long period.

\section{Facial Expressions Module}

Human beings easily read others' feelings from their facial expressions because when they have some feelings for some situation in which they are put in, they make facial expressions reflecting their feelings. Therefore, it is not too much to say that an agent with facial expressions makes human easy to understand the situations.

In order to give a partner player friendliness and a better understanding of game situations, facial expressions are introduced to the partner system. To construct models expressing the relationship between facial expressions and game situations, neural network models are used in this paper.

1) Construction of Facial Expressions Model

Fig. 3 shows an example of the neural network model. Inputs of the neural network model are 8 factors expressing game situations, result in Eq. (4), the number of turn, potential in Eq. (5), current hand score which is the expected value of the current human player's hand score, opponent's bet, change of result which is the difference between result of the current situation and result of the previous situation, availability of bluff and availability of slow play which have the value 1 or 0 according to Eq. (10) and Eq. (11), respectively.

$$
\begin{aligned}
& \text { availability of bluff }=\left\{\begin{array}{l}
1 \text { : Eq. (6) is satisfied } \\
0: \text { otherwise }
\end{array}\right. \\
& \text { availability of slow play }=\left\{\begin{array}{l}
1 \text { : the human player's hand } \\
\text { is hidden in hole cards } \\
0: \text { otherwise }
\end{array}\right.
\end{aligned}
$$

The degree of eyebrow lean, the form of eyebrow, the degree of eye lean, the form of eye, the size of eyes, the form of an upper lip, the form of a lower lip and the width of a mouth, are considered as outputs of neural network models, which are called facial features values in this paper. In this paper, facial expressions are generated by 8 facial features values, where neural network models abovementioned. There are 8 neural network models with an output, because structures of a neural network model with an output are simple. These neural network models have an input layer, a hidden layer with twenty nodes and an output layer.

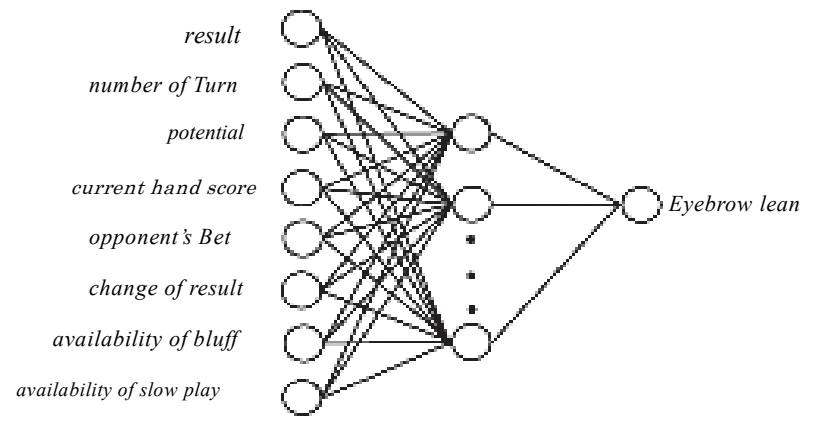

Fig. 2. Example of neural network model

\section{2) Facial Feature Values}

As mentioned before, this paper uses 8 kinds of facial feature values. And these facial features values are expressed by the coordinates values of 20 points of facial features as shown in Fig. 4, where, it is assumed that the origin of the coordinates is set as shown in Fig .4. Facial feature values are obtained as follows.

The degree of eyebrow lean and the form of eyebrow are defined by Eqs. (12) and (13), respectively, where the $y$-coordinate values of both ends of eyebrow are $e b 1 y$ and $e b 2 y$, and the $y$-coordinate value of the center position of eye brow is $e b 3 y$.

Eyebrow lean: $e b 1_{y}-e b 2_{y}$

Form of eyebrow: $e b 3_{y}-\left(e b 1_{y}+e b 2_{y}\right) / 2$

In the same way, the degree of eye lean and the form of eye is obtained by Eqs. (14) and (15), respectively, where the $y$-coordinate values of both ends of eye are $e 1 y$ and $e 2 y$, and the $y$-coordinate value of the center position of eye is $e 3 y$.

Lean of eye: $e 1_{y}-e 2_{y}$

Form of eye: $e 3_{y}-\left(e 1_{y}+e 2_{y}\right) / 2$

The size of eye is defined by Eq. (16), where both ends of eyes are $e 4 y$ and $e 5 y$.

Size of eye: $e 4_{y}-e 5_{y}$

The width of a mouth is defined by Eq.(17), where the $x$-coordinate values of both ends of a mouth are $m 1 x$ and $m 2 x$.

Width of lip: $m 2_{x}-m 1_{x}$

The form of an upper lip and that of a lower lip are obtained by Eqs. (18) and (19), respectively, where the $y$-coordinate value of the center of an upper lip is $m 3 y$ and that of a lower lip is $m 4 y$.

Form of upper lip: $m 3_{y}-m 1_{y}$

Form of lower lip: $m 4_{y}-m 1_{y}$ 
Parameter values about the size, the lean and the width are normalized as $0.0-1.0$, the minimum value - the maximum value.

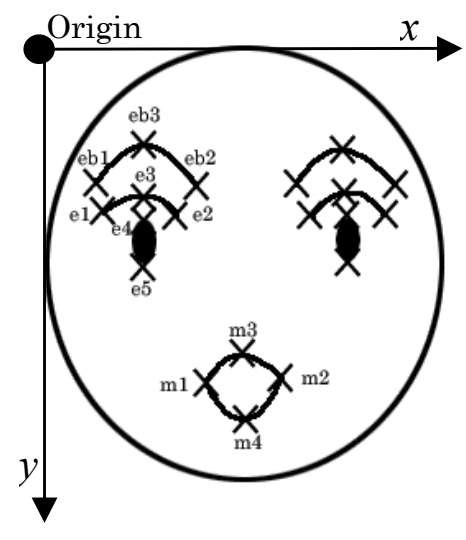

Fig. 3. Facial features points

\section{Cooperation Module}

The cooperation section has two parts. One part gives a human partner player strategy to take in the current game situation and its grounds. The other gives a human player reply to human player's questions about the current game situation.

\section{1) Strategy Presentation}

System's strategy presentation to a human partner player is performed at the decision-making process in each betting round or at the process of the human player's choice of a hole card to be revealed after the fifth turn. When the partner system is called by a human player, the system gives strategy for the current game situation.

\section{a) Decision to fold or not to fold}

At the beginning of each betting round, the partner system presents its decision and its grounds to a human player according to the assessment of superiority/inferiority obtained by Eq. (4). If the system decides to fold according to III.B.3), then the system presents its grounds to a human player.

\section{b) Decision of amount of bed}

The partner system presents the point value obtained by III.B.4). For example, when the system decides the betting point to be 10 , the system says that 10 points should be bet for the current situation.

\section{c) Decision to call}

After an opponent player bets some points, these decision procedures are performed. The system decides to fold or not as the same procedures in $a$ ).

\section{d) Choice of a card to be revealed}

The system presents a card chosen by the procedure in III.B.5).

\section{e) A human player takes another strategy}

At the situation of decision to fold, although the system presents strategy to fold a game to a human player, if a human player tries to take different strategy from the presented one, the system calls the attention to a human player about the game continuation. For example, the system presents "You should fold, because your hand is weak and opponent player's bet is large".

2) Answer to Player's Question

When a human partner player asks the system some question about the current game situation, the system replies to it. The following question items are considered in this paper: Human player's game playing tendency, estimated opponent player's hand strength, estimated human player's expected hand, estimated human player's hand strength from the opponent player's side.

\section{a) Human player's game playing tendency}

When the system gets a question about human player's game playing tendency, the system presents linguistic expressions based on the tendency value $t_{i j}$ explained in III.B.2) Although the tendency value $t_{i j}$ is essentially obtained for an opponent player, the partner system can obtain not only an opponent player's tendency value but also a partner player's one by the procedures described in III.B.6). For example, the system says that a human partner player tends to be passive in a situation such as the current situation. TABLE III shows the linguistic expressions corresponding to $t_{i j}$.

TABLE III

LINGUISTIC EXPRESSIONS CORRESPONDING TO $t_{i j}$

\begin{tabular}{|c|c|}
\hline$t_{i j}$ & Linguistic Expressions \\
\hline $100-70$ & Very aggressive \\
\hline $70-60$ & Aggressive \\
\hline $60-50$ & Rather aggressive \\
\hline $50-40$ & Rather passive \\
\hline $40-30$ & Passive \\
\hline $30-0$ & Very passive \\
\hline
\end{tabular}

b) Estimated expected opponent player's hand

The system performs simulation repeatedly by assigning possible combination of cards to opponent player's hole cards and/or opponent player's cards dealt from now on. Then, the system obtains the opponent player's poker hand with the highest hand probability and the poker hand with the highest score with its probability. The system presents these poker hands and their estimated probabilities to a human partner player. For example, the system says that the opponent player's hand with the highest probability is One Pair with $46.9 \%$ and that the estimated highest score hand is Straight with $10.5 \%$. If a human partner player asks the system about other poker hands, the system also replies them. 
c)

\section{Estimated expected human player's hand}

The system performs simulation repeatedly by assigning possible combination of cards to a human partner player's cards dealt from now on, and obtains the partner player's poker hand with the highest hand probability and the poker hand with the highest score with its probability. Then, the system presents these poker hands and their estimated probabilities to a partner player. If a human partner player asks the system about other poker hands, the system also replies them.

\section{d) Estimated human player's hand strength from opponent player's side}

In the same way as $b$ ), the system performs simulation repeatedly by assigning possible combination of cards to a human partner player's hole cards and to cards to be dealt from now on, and obtains the partner player's poker hand with the highest hand probability and the highest score poker hand with its probability. Then, the system presents these poker hands and their estimated probabilities to a partner player. If a human partner player asks the system about other poker hands, the system also replies them.

\section{EXPERIMENTS}

\section{A. Construction of Facial Expressions Model}

Neural network models expressing the relationship between facial expressions and 8 factors expressing game situations are obtained for each subject using each subject's own data and the back propagation method. Subjects draw facial expressions according to the situation of the seven-card stud poker game using the human interface as shown in Fig. 5. Subjects draw the facial expression by adjusting the parameter values using the buttons on this interface. Eight factors values expressing the current game situation are obtained by calculation of, e.g., Eq. (4) and Eq (5). Facial feature points are obtained by facial expressions drawn by subjects. And facial features values such as the size, the width and the lean are obtained by Eqs. (12) - (19) and are normalized in $[0,1]$. These values are used as training data for neural network models learning. Thirty-three training data sets are collected for each subject. After the learning the questionnaire is performed in order to confirm whether obtained neural network models generate facial expressions reflecting poker game situation or not. Each subject evaluate facial expressions with a 5-pont scale: The model generates facial expressions, 5: very well, 4: well, 3: neutral, 2: badly, 1:very badly. As for questionnaire results, five out of 8 marks 4 and the average score among all subjects is 3.5. It is found that obtained neural network models express facial expressions reflecting poker game situations a little well.

\section{B. Experimental Procedures}

Subject experiments are carried out in order to confirm the usefulness and friendliness of the presented system. Each subject plays 20 poker games with the presented partner system with facial expressions which obtained by III. 1) against the poker playing system and other 20 poker games with previous partner system without facial expressions [14]. In the experiments two kinds of card sets, $\alpha$ and $\beta$, are prepared. Subjects are divided into two groups in order to analyze playing strategy in every game with/without facial expressions. Subjects in one group play a poker game using card set $\alpha$ with facial expressions and play it using card set $\beta$ without facial expressions. Subjects in other group play a poker game using reversed card sets. Cards in each card set are dealt to all subjects in the same order. That is, all subjects play games on the same condition in the experiments. And if all 20 games are finished by a showdown, prepared cards in card sets $\alpha$ and those in card set $\beta$ are set to have 10 wins and 10 loses, respectively. However, subjects do not know them.

The number of subjects is 8 . Although they are novices of a poker game, they have knowledge on poker game rules. Subjects A, B, C, and D play a poker game using a card set $\alpha$ with facial expressions. Subjects E, F, G, and H play a game using a card set $\beta$ with facial expressions. And subjects A, C, $\mathrm{E}$, and $\mathrm{G}$ play a game using a card set $\alpha$ at the beginning. Subjects B, D, E, and H play a game using a card set $\beta$ at the beginning.

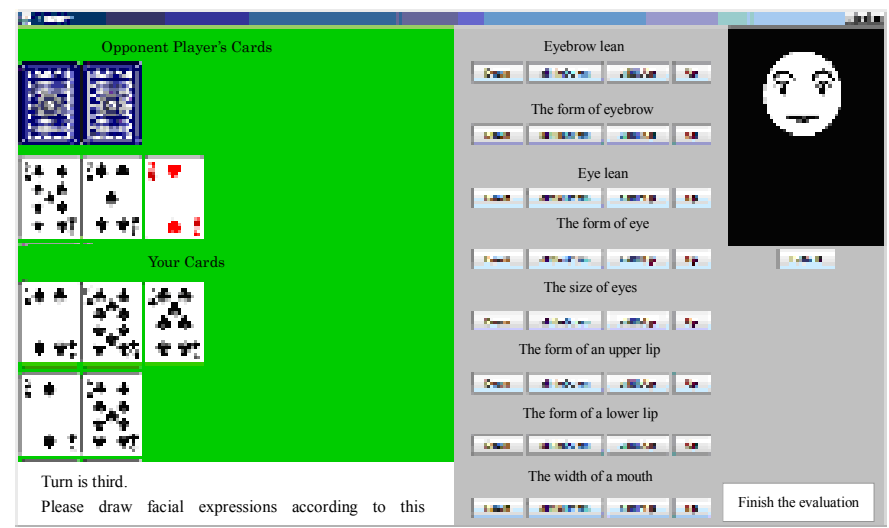

Fig. 4. Example of human interface for facial expressions model

\section{Experimental Results \\ 1) Gain/Loss of subjects}

TABLE IV shows the overall gain/loss points of each subject with/without facial expressions against the playing system, where shaded parts show that subjects play a game with facial expressions. Paired T-tests of gain/loss points of all subjects with/without facial expressions are carried out. The value of t-distribution with degree of freedom 8 is -0.28 and significance level $5 \%$ is 2.365 . The null hypotheses are not rejected. This test shows that there is a no difference between gain/loss points with the facial expressions and those without facial expressions. 


\section{2) Evaluations of partner system}

\section{a) Usefulness of partner system}

After the experiments subjects evaluate the usefulness of the partner system with a 5-pojnts scale, 5: very useful, 4: useful, 3: neutral, 2: useless, 1: very useless. Fig. 6 shows the evaluation results. Four subjects out of 8 mark 4 or 5 and the average score among all subjects is 3.75 , more or less useful. And there are no subjects marking 2 or 1 . Then subjects' evaluation of the partner system is more or less high. As a mater of fact, some subjects have comments that the partner system is useful for the estimation of opponent player's hand and the estimation of superior/inferior of the partner player's hand.

TABLE IV

GAIN/LOSS OF SUBJECTS

\begin{tabular}{|c|c|c|}
\multicolumn{2}{c}{ GAIN/LOSS OF SUBJECTS } \\
\cline { 2 - 3 } Subject & \multicolumn{2}{|c|}{ Gain/Loss of Subjects } \\
\hline Cards set $\alpha$ & Cards set $\beta$ \\
\hline B & -41926 & 42355 \\
\hline C & -14996 & 27532 \\
\hline D & 60063 & 45743 \\
\hline E & 13087 & 8543 \\
\hline F & 28042 & 91397 \\
\hline G & 22269 & 65852 \\
\hline H & -86228 & -21047 \\
\hline
\end{tabular}

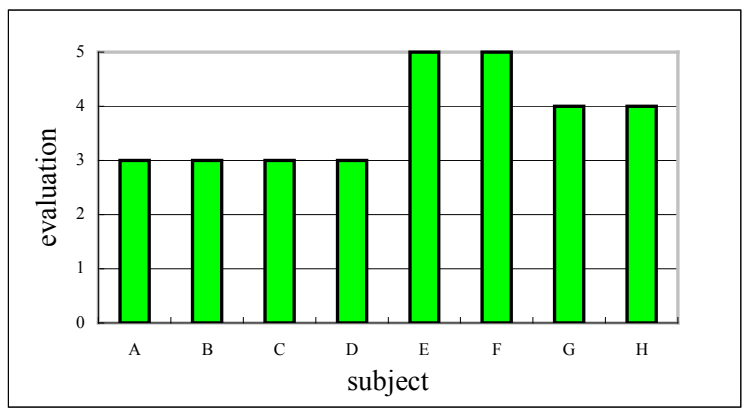

Fig. 5. Usefulness of partner system

\section{b) Friendliness of partner system}

Subjects evaluate the friendliness of the partner system with facial expressions compared to the partner system without facial expressions with a 5-points scale, 5: very friendly, 4: friendly, 3: neutral, 2: unfriendly, 1: very unfriendly. Fig. 7 shows the evaluation results. Six subjects out of 8 mark 4 or 5 and the average score among all subjects is 4 . It is found that subjects' evaluation of friendliness of the partner system with facial expressions is high.

Some subjects have comments that they feel that they share in their joys for winning a game or in their sorrows for losing a game with the partner system. Some other subject also shows the following comments: The subject evaluates the partner system with facial expressions very friendly from the following reasons. Although the partner system's linguistic expression is your hand is rather strong, facial expressions seems not so happy. Then, the subject feels that these expressions mean it is difficult for the system to estimate the current situation. It is found that this subject has delicate feelings from linguistic expression with facial expressions. Fig. 8 shows the facial expressions presented to this subject in this situation. Some other subject evaluates the partner system with facial expressions friendly because this subject feels loneliness when he plays a game with the partner system without facial expressions.

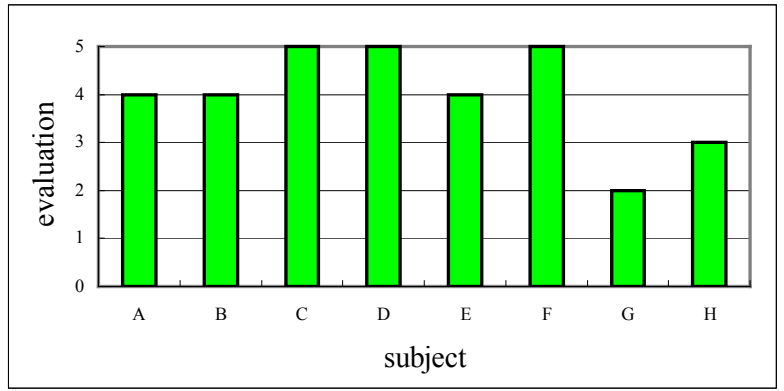

Fig. 6. Friendliness of partner system

\section{c) Change of facial expressions}

Fig. 9 shows an example of facial expressions changes according to situations by subject $\mathrm{H}$. First, the partner system presents: In this situation, hand is very weak, but turn is only the fourth. So, you should not fold Facial expressions look rather sad. However, subject H's hand becomes strong in the next turn. The partner system presents: In this situation, hand is rather strong. So, you should not fold. Facial expressions look surprise. When subject $\mathrm{H}$ bets some points, the partner system presents: In this situation, hand is strong. So, you should bet 1022 points. And facial expressions look happy.

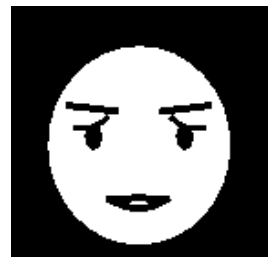

Fig. 7. Example of facial expressions

\section{CONCLUSIONS}

This paper constructs the partner system that supports a human player in playing a seven-card stud poker game. This system presents its own strategy, facial expressions and/or replies some questions about game situations.

Subject experiments are performed to confirm the usefulness and the friendliness of the presented partner system with facial expressions. Subject's evaluations of the friendliness of the partner system with facial expressions are high. 


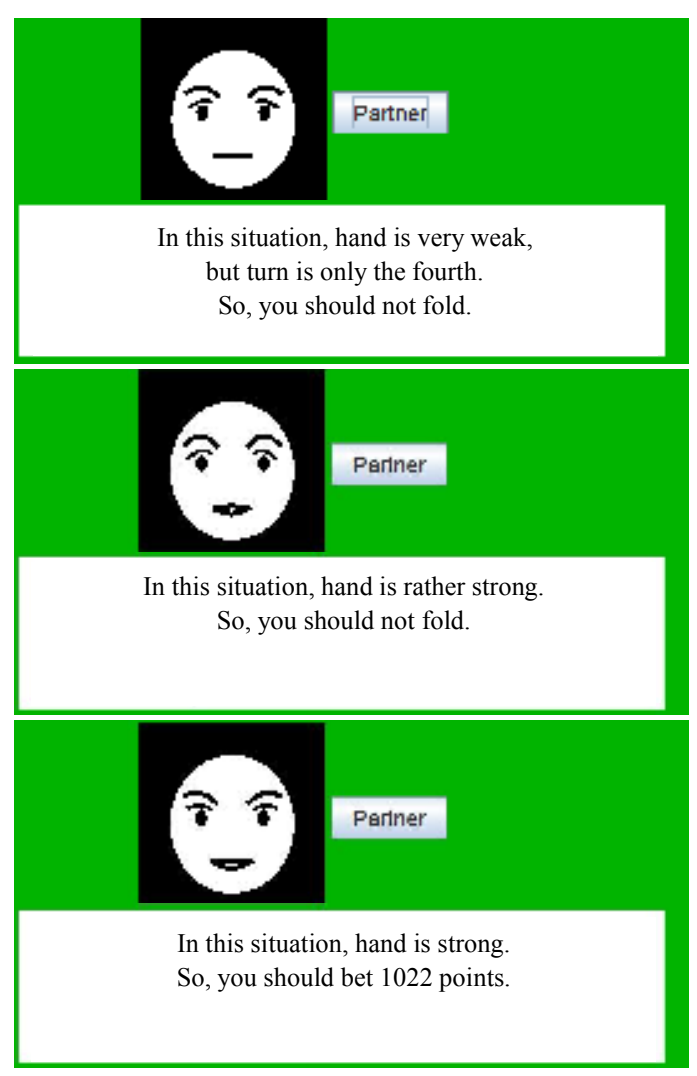

Fig. 8. Example of change of facial expressions

\section{REFERENCES}

[1] Toshimasa Moriwaki: "group, organization" Tokyo Daigaku Shuppan, 2000

[2] Koki Matsura: "Negotiation Simulation System Based on Fuzzy Decision-Making" Journal of Japan Society for Fuzzy Theory and System, Vol.8, No.5,pp882-890 1996

[3] Nobuyuki NAKAJIMA, Motomasa DAIGO, YukoYAMASHITA: "Analysis and Construction of Fuzzy Purchase Decision Support System" Journal of Japan Society for Fuzzy Theory and System, Vol.8 No.5, pp871-881, 1996
[4] Gleb Beliakov and Jim Warren, "Appropriate Choice of Aggregation Operators in Fuzzy Decision Support System" IEEE TRANSACTION ON FUZZY STSTENS, vol. 9, No. 6, 2001 pp.773-783

[5] Norio Sasaki: "Introduction of game theory"Nippon Hyoron Sha, 2003

[6] Toshiaki Yasumura, Hidehisa Akiyama, Kunihiko Oguti, Katsumi Nitta: "Negotiation Agents in the MONOPOLY Game" Information Processing Society Japan Vol43, No.10, pp3048-3055, 200

[7] Darse Billings, Aron Davidson, Jonathan Schaeffer, Duane Szafron, "The challenge of poker" Artificial Intelligence 134(2002) , pp.201-240

[8] Graham Kendall, Mark Willdig, "An Investigation of an Adapting Poker Player" Artificial Intelligense 2001, LNAI 2246, pp.189-200

[9] Arild Sandven and Bjorner Tessem, "A Case-Based Learner for Poker" Proceedings of the Ninth Scandinavian Conference on Artificial Intelligence (SCAI 2006) pp. 159-167

[10]A. Meharabian, "Silent messages: Implicit communication of emotions and attributes", $2^{\text {nd }}$ ed., Wadsworth, California, 1980.

[11] Koda T, Maes P, "Agents with faces: the effect of personification" Robot and Human Communication, 1996., 5th IEEE International Workshop pp. 189-194

[12] Takehisa ONISAWA, Satoru KAZAMI, Chiharu TAKAHASHI, "Construction of Playing System of Game with Imperfect Information -Seven-Card Stud Poke-”, Journal of Japan Society for Fuzzy Theory and System, Vol.15,No.1,pp127-141,2003

[13] T. Onisawa and C.Takahashi: Poker Playing system Considering Opponent Player's Strategy, Proceedings of SICE Annual Conference 2004 in Sapporo, pp.2347-2352, 2004

[14] K. Ohsone, T. Onisawa "Cooperative Partner System of Imperfect Information Game" 2008 IEEE Conference on Soft Computing in Industrial Applications (SMCia/08), June 25-27, 2008, Muroran, JAPAN pp. 60-65

[15]R. Kruse, J. Gebhardt, F.Klawonn "Foundations of FUZZY SYSTEMS" JOHN WILEY \& SONS, 1994 